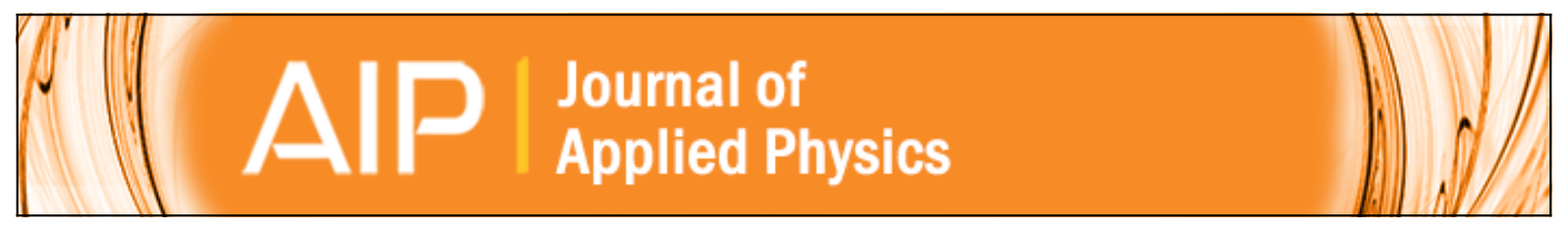

Damage accumulation in Si crystal during ion implantation at elevated temperatures: Evidence of chemical effects

J. P. de Souza, Yu. Suprun-Belevich, H. Boudinov, and C. A. Cima

Citation: Journal of Applied Physics 87, 8385 (2000); doi: 10.1063/1.373551

View online: http://dx.doi.org/10.1063/1.373551

View Table of Contents: http://scitation.aip.org/content/aip/journal/jap/87/12?ver=pdfcov

Published by the AIP Publishing

Alp Re-register for Table of Content Alerts

Create a profile. 


\title{
Damage accumulation in Si crystal during ion implantation at elevated temperatures: Evidence of chemical effects
}

\author{
J. P. de Souza, Yu. Suprun-Belevich, ${ }^{\text {a) }}$ and H. Boudinov \\ Instituto de Física, Universidade Federal do Rio Grande do Sul, 91501-970 Porto Alegre, R.S., Brazil \\ C. A. Cima \\ PGCIMAT, Universidade Federal do Rio Grande do Sul, 91501-970, Porto Alegre, R.S., Brazil
}

(Received 2 September 1999; accepted for publication 28 February 2000)

\begin{abstract}
Damage in Si induced by irradiation with various light/medium mass ions at elevated temperatures and high doses $\left(\geqslant 3 \times 10^{16} \mathrm{~cm}^{-2}\right)$ was studied using Rutherford backscattering spectroscopy, cross-section transmission electron microscopy, and high resolution x-ray diffraction. The results obtained have shown that there is a marked variation in the damage accumulation for different ion species. For $\mathrm{O}^{+}$and $\mathrm{N}^{+}$ions a distinct layer with a low level of damage presenting negative strain is formed at the surface. It has been found that the magnitude of the strain does not correlate with the energy deposited in the collision cascades. In the cases of $\mathrm{Ne}^{+}$and $\mathrm{Mg}^{+}$implantation, a low damage accumulation occurs near the surface but no negative strain is formed. In contrast to the $\mathrm{N}^{+}$and $\mathrm{O}^{+}$cases, with the increase of the $\mathrm{Ne}^{+}$or $\mathrm{Mg}^{+}$dose $\left(>1 \times 10^{17} \mathrm{~cm}^{-2}\right)$ the damage profile stretches almost to the crystal surface. It is proposed that in addition to the mechanism of spatial separation of Frenkel pairs taking place in the collision cascades, the ability of the implanted ions to form precipitates and complexes with $\mathrm{Si}$ atoms noticeably influences the damage formation during implantation at elevated temperatures. (c) 2000 American Institute of Physics.

[S0021-8979(00)02112-5]
\end{abstract}

\section{INTRODUCTION}

It is generally accepted that residual damage in ion implanted crystals decreases with the increasing temperature of the implantation. Below $\sim 200^{\circ} \mathrm{C}$ the damage accumulation for medium and heavy mass ions eventually results in formation of an amorphous layer at sufficiently high doses. However, above this temperature the crystalline-toamorphous transformation does not take place, irrespective of the dose. ${ }^{1,2}$ High dose implantation at elevated temperatures has been used to synthesize a variety of compounds in silicon including silicon dioxide, silicon nitride and metal silicide buried layers. ${ }^{3}$ Ion implantation at high temperatures provides the possibility of exploring damage-related phenomena at very high doses $\left(>10^{17} \mathrm{~cm}^{-2}\right) \cdot{ }^{2-6}$ Understanding of the mechanisms for damage growth at high dose ion implantation at elevated temperatures is of great importance for technologies for synthesis of silicon on insulator (SOI) materials.

It was previously demonstrated that implantation of $\mathrm{Si}^{+}$ and $\mathrm{O}^{+}$ions into $\mathrm{Si}$ crystal at elevated temperatures leads to the formation of two distinctly different layers: a heavily dislocated region near the ion range, and a shallower layer which is dislocation free but shows a mechanical strain of contraction. ${ }^{2,4,7}$ The mechanical strain is induced by the vacancy clusters formed from the surplus vacancies accumulated at the surface. This effect was supposed to be intrinsic to the conditions of the implantation (i.e., high temperature and dose) rather than dependent on the ion-solid chemistry. ${ }^{2}$ The role of the impurity atoms in the growth of the damage

\footnotetext{
${ }^{a)}$ Electronic mail: belevich@if.ufrgs.br
}

is not yet clear at present. There is no detailed information available on the damage growth during implantation of $\mathrm{Si}$ with ions different from $\mathrm{O}^{+}$and $\mathrm{Si}^{+}$at elevated temperatures.

In this article, we present the results of a comparative investigation of damage growth at high temperature implantation of $\mathrm{Si}$ with different ions of light or medium mass elements. The influence of the implanted species on the process of the formation of a layer with negative strain and on the damage accumulation has been studied.

\section{EXPERIMENT}

Czochralski-grown silicon wafers of $n$-type conductivity, with resistivity of $1-2 \Omega \mathrm{cm}$ and (100) orientation, were implanted at $600{ }^{\circ} \mathrm{C}$ with $120 \mathrm{keV}{ }^{14} \mathrm{~N}^{+}$, 90 or $120 \mathrm{keV}{ }^{16} \mathrm{O}^{+}$, $140 \mathrm{keV}{ }^{20} \mathrm{Ne}^{+}$and $150 \mathrm{keV}{ }^{24} \mathrm{Mg}^{+}$ions to doses in the range of $0.3-2.0 \times 10^{17} \mathrm{~cm}^{-2}$. The beam current density was maintained in the range of 3-6 $\mu \mathrm{A} \mathrm{cm}^{-2}$. In order to minimize channeling effects during the implantation, the samples were tilted $7^{\circ}$ and rotated $25^{\circ}$ with respect to the $\langle 110\rangle$ direction.

The implanted samples were analyzed by Rutherford backscattering (RBS) spectrometry using an incident beam of $900 \mathrm{keV}{ }^{4} \mathrm{He}^{++}$ions in random and in $\langle 100\rangle$ crystal directions. The depth profiles of the damage were obtained from the RBS spectra using an iterative calculation procedure with the two dechanneled beam approximation. ${ }^{8}$

Internal mechanical strain in the samples was investigated by high-resolution $\mathrm{x}$-ray diffraction measurements in double-axis and triple-axis configurations using a $\mathrm{Cu} K \alpha_{1}$ beam. By measuring the angular distance between the dif- 


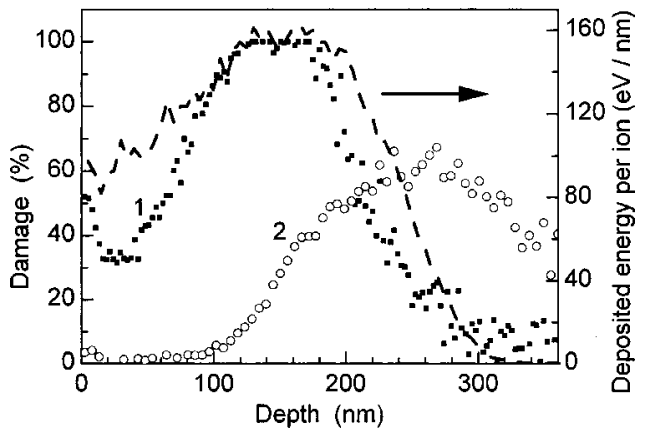

FIG. 1. Damage profiles extracted from the RBS spectra from Si implanted with $90 \mathrm{keV} \mathrm{O}^{+}$ions. (1) Room temperature implantation dose of $2 \times 10^{15}$ $\mathrm{cm}^{2}$; (2) implantation at $600{ }^{\circ} \mathrm{C}$, dose of $7.5 \times 10^{16} \mathrm{~cm}^{-2}$. The energy deposited in nuclear collisions calculated by TRIM is shown by the dashed curve.

fraction peaks from the Si substrate and the implanted layer in the (004) orientation in the rocking curves, the relative perpendicular mismatch $(\Delta d / d)_{\perp}$ was obtained. The in-plane component of the strain was found to be negligible by examination of the reflections from the inclined lattice planes. To obtain additional information about the depth distribution of the strain the measurements were repeated after the top layer of the samples had been removed by etching.

A limited set of the implanted samples was investigated also by cross-section transmission electron microscopy (TEM).

\section{RESULTS AND DISCUSSION}

The damage profiles calculated from the experimental RBS spectra from $\mathrm{Si}$ implanted with $\mathrm{O}^{+}$ions at room temperature (RT) and at $600^{\circ} \mathrm{C}$ along with the profile for the energy deposited in nuclear collisions simulated by TRIM ${ }^{9}$ are presented in Fig. 1. There, 100\% of the damage indicated corresponds to the "random level" or 100\% dechanneling. The following features are disclosed in Fig. 1. As is usually observed, the damage profile after RT implantation closely correlates to the distribution of the energy deposited in nuclear collisions, estimated by TRIM. There is a $50 \mathrm{~nm}$ thick buried amorphous layer centered at a depth of $140 \mathrm{~nm}$ after RT implantation. However, the damage distribution in the sample implanted with $\mathrm{O}^{+}$ions at $600{ }^{\circ} \mathrm{C}$ is located $\sim 120$ $\mathrm{nm}$ deeper than in the sample implanted at RT. After high temperature implantation a layer free of the damage responsible for dechanneling is formed in the region extending from the surface up to a depth of $\sim 100 \mathrm{~nm}$. It can be seen that the damage distribution in this case shows no correlation to the TRIM distribution of the energy deposited in nuclear collisions.

The damage accumulation and distribution observed in the case of high temperature $\mathrm{O}^{+}$implants are in accordance with the results reported in the literature. ${ }^{4,7}$ There are two distinct damage layers in the samples. A buried damaged layer is located around the projected range of ions, and a second layer, which is supposed to have a unidirectional strain of lattice contraction, is formed at the crystal surface. ${ }^{4,7}$ The RBS studies of the samples implanted at elevated temperatures within the dose range investigated

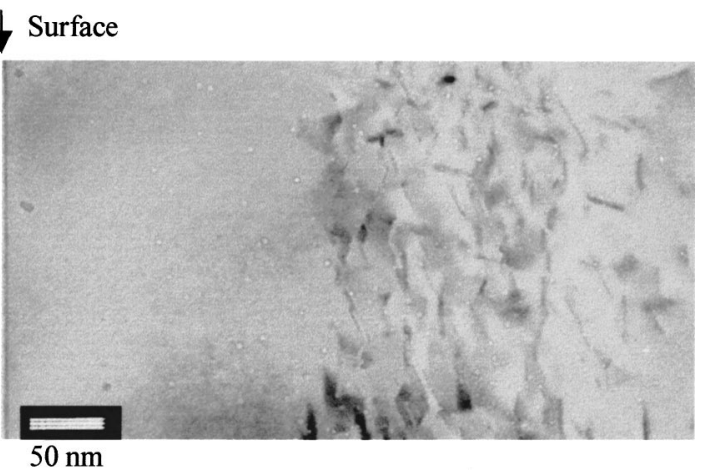

FIG. 2. Cross-section TEM micrograph of Si implanted with $140 \mathrm{keV} \mathrm{Ne}$ ions at $600^{\circ} \mathrm{C}$, dose of $1 \times 10^{16} \mathrm{~cm}^{-2}$. Small white spots represent precipitates (bubbles).

showed no amorphous layer. Therefore, the damage distribution observed is not a result of a solid phase epitaxy process. This damage consisting of extended defects is created during the high temperature implantation. The level of the damage (dechanneling) observed after the implantation correlates with the data reported in the literature. ${ }^{2,4}$ The dechanneling of $\mathrm{He}^{++}$ions in the surface layer is negligible, showing almost no damage in this region on the profiles extracted from the RBS spectra. Similar results were reported also for $\mathrm{Si}$ implanted with $\mathrm{Si}^{+}$ions. ${ }^{2,5}$ A mechanism based solely on the kinematics of the ion-atom interaction (and not the chemistry) was proposed to account for the presence of these two layers in self-implanted $\mathrm{Si}^{2}{ }^{2,5}$ The separation of these two layers was suggested to be due to a spatial separation of Frenkel interstitial-vacancy pairs created during irradiation as a result of atomic displacement. ${ }^{2,5}$ The momentum transferred to the interstitial $\mathrm{Si}$ atom should have a nonzero component along the direction of the incident ion. This should lead to spatial separation between the interstitial and vacancy defects with the interstitials distributed to a greater depth on average. Thus, vacancies mainly located at the surface form vacancy type defect clusters, which induce the negative strain observed, and interstitials promote the formation of extended defects detected by the RBS around the projected range of the ions. The suggested explanation ${ }^{2}$ was supported by the results of calculations of vacancy and interstitial distributions using a modified version of TRIM. ${ }^{10}$

A typical cross-section TEM micrograph of the sample implanted with $140 \mathrm{keV} \mathrm{Ne}^{+}$ions to a dose of $1 \times 10^{16} \mathrm{~cm}^{-2}$ at $600^{\circ} \mathrm{C}$ is shown in Fig. 2. This micrograph was compared with those of the $\mathrm{O}^{+}$-implanted samples (not shown here, since similar micrographs have been published elsewhere ${ }^{6,7}$ ). None of the TEM analyses of the samples implanted at high temperatures showed any defects in the near surface region except for the $\mathrm{Ne}^{+}$-implanted samples. In the $\mathrm{Ne}^{+}$-implanted samples precipitates (bubbles) represented by small white spots (Fig. 2) are found in the near surface region and in the depth near $R_{p}$. Apart from this, no significant difference is observed by the TEM analyses for the samples investigated. The vacancy clusters formed in the near surface region, that would account for lattice contraction, are not visible by TEM due to their small size. There is a band of extended defects at a depth of $150-350 \mathrm{~nm}$ (that is, near $R_{p}$ ) with a sharp border 


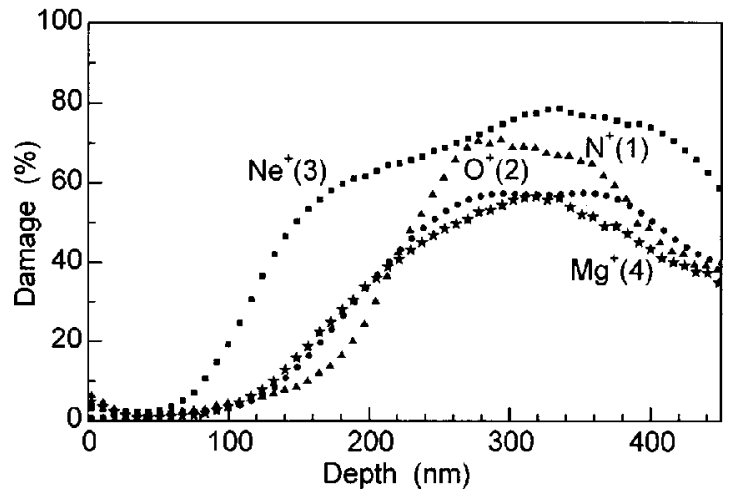

FIG. 3. Damage profiles extracted from the RBS spectra. Implantation at $600{ }^{\circ} \mathrm{C}$ : (1) $120 \mathrm{keV} \mathrm{N}^{+}, 2 \times 10^{17} \mathrm{~cm}^{-2}$; (2) $120 \mathrm{keV} \mathrm{O}^{+}, 1.5 \times 10^{17} \mathrm{~cm}^{-2}$; (3) $140 \mathrm{keV} \mathrm{Ne} \mathrm{Ne}^{+} 10^{17} \mathrm{~cm}^{-2}$; (4) $150 \mathrm{keV} \mathrm{Mg}{ }^{+}, 3 \times 10^{16} \mathrm{~cm}^{-2}$.

with the near surface damage free region. No amorphous layer was observed by TEM in the samples implanted at high temperatures.

Figure 3 shows the damage profiles calculated from the RBS spectra from $\mathrm{Si}$ implanted with $\mathrm{N}^{+}\left(2 \times 10^{17} \mathrm{~cm}^{-2}\right.$ at $120 \mathrm{keV}), \mathrm{O}^{+}\left(1.5 \times 10^{17} \mathrm{~cm}^{-2}\right.$ at $\left.120 \mathrm{keV}\right), \mathrm{Ne}^{+}\left(1 \times 10^{17}\right.$ $\mathrm{cm}^{-2}$ at $\left.140 \mathrm{keV}\right)$ and $\mathrm{Mg}^{+}\left(3 \times 10^{16} \mathrm{~cm}^{-2}\right.$ at $\left.150 \mathrm{keV}\right)$ ions at $600^{\circ} \mathrm{C}$. The projected ranges of the ions in these cases are $\sim 260 \mathrm{~nm}$. The doses of $\mathrm{N}^{+}, \mathrm{O}^{+}$and $\mathrm{Ne}^{+}$ions were chosen to produce approximately the same estimated values of the energy deposited in nuclear collisions. The damage profiles in Fig. 3 should not be compared with the profiles in Fig. 1 because the corresponding implantation energies were different. The data presented in Fig. 3 demonstrate that similar to the case of $\mathrm{O}^{+}$implantation the damage distributions in the samples implanted at high temperature with $\mathrm{N}^{+}, \mathrm{Ne}^{+}$and $\mathrm{Mg}^{+}$ions are shifted towards the end of range of the ions while a $\sim 100 \mathrm{~nm}$ thick layer with low damage is formed near the surface. In the cases of $\mathrm{O}^{+}$and $\mathrm{N}^{+}$implantation, the damage profile remains almost unchanged for doses from 3 $\times 10^{16}$ to $2 \times 10^{17} \mathrm{~cm}^{-2}$, indicating saturation of the damage accumulation. However, the increase of the dose of $\mathrm{Ne}^{+}$or $\mathrm{Mg}^{+}$ions from $3 \times 10^{16}$ to $1 \times 10^{17} \mathrm{~cm}^{-2}$ leads to broadening of the damage profile, mostly towards the surface.

Typical rocking curves of the samples investigated are shown in Fig. 4. The rocking curves from the samples implanted with $\mathrm{O}^{+}$and $\mathrm{N}^{+}$ions show large substrate reflection and a secondary peak to the right of the main peak. This secondary peak indicates that there is a region in the samples with negative lattice strain (contraction). The maximum magnitude of the strain $\left(1.4 \times 10^{-3}\right)$ was obtained for the sample implanted with $\mathrm{N}^{+}$ions at a dose of $2 \times 10^{17} \mathrm{~cm}^{-2}$. The rocking curves from the samples implanted with $\mathrm{Ne}^{+}$ and $\mathrm{Mg}^{+}$ions do not have any secondary peaks, they only show a small asymmetry to the left of the main peak from the substrate indicating presence of some low positive strain (expansion) in the sample. To obtain the position of the region with negative strain along the depth of the samples implanted with $\mathrm{O}^{+}$and $\mathrm{N}^{+}$ions, the surface layer with a thickness of about $100 \mathrm{~nm}$ was removed by chemical etching. The x-ray measurements following the removal showed no negative strain, indicating that the region with negative

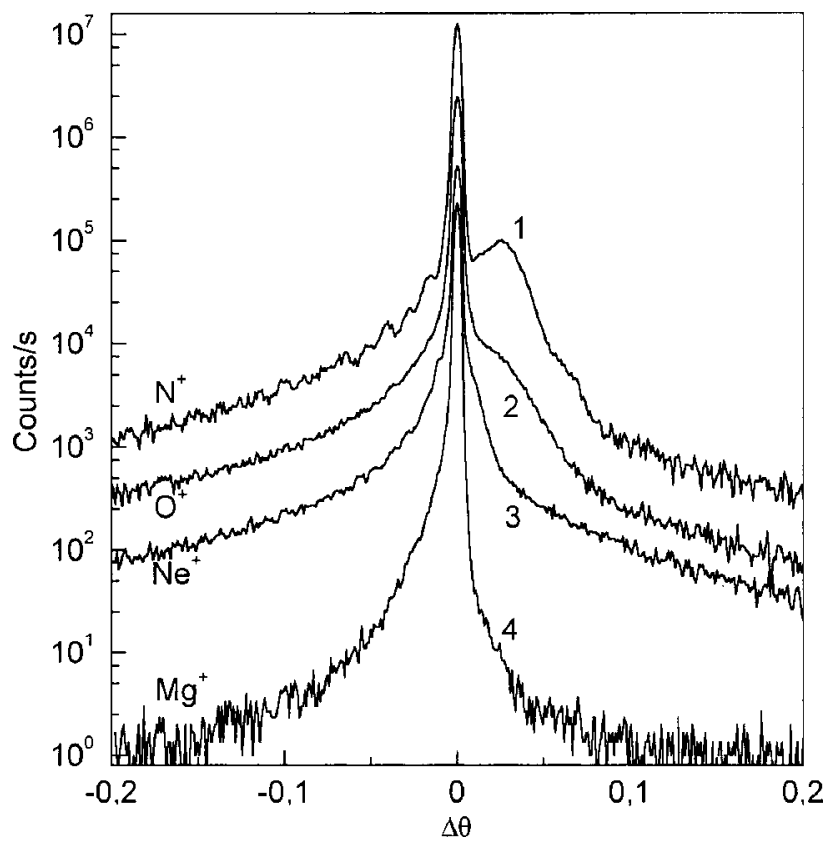

FIG. 4. X-ray rocking curves from $\mathrm{Si}$ implanted at $600^{\circ} \mathrm{C}$ with (1) $120 \mathrm{keV}$ $\mathrm{N}^{+}, 10^{17} \mathrm{~cm}^{-2}$; (2) $120 \mathrm{keV} \mathrm{O}^{+}, 10^{17} \mathrm{~cm}^{-2}$; (3) $140 \mathrm{keV} \mathrm{Ne}+10^{17} \mathrm{~cm}^{-2}$; (4) $150 \mathrm{keV} \mathrm{Mg}^{+}, 3 \times 10^{16} \mathrm{~cm}^{-2}$.

strain is located near the surface of the sample. According to the RBS studies, this region does not cause dechanneling. High temperature annealing (e.g., $1200^{\circ} \mathrm{C}$ ) completely eliminates the negative strain in the samples irradiated with $\mathrm{O}^{+}$or $\mathrm{N}^{+}$ions. Since $\mathrm{N}$ has a lower mass than $\mathrm{O}$, the $\mathrm{N}^{+}$ ions were expected to produce lower negative strain due to a lower number of Frenkel pairs created. In fact, the negative strain observed in the case of $\mathrm{N}^{+}$implantation is essentially higher than that in the case of $\mathrm{O}^{+}$implantation (see Fig. 4). Completely different mechanical strain behavior was observed in Si implanted with $\mathrm{Ne}^{+}$and $\mathrm{Mg}^{+}$ions (see Fig. 4). These facts clearly indicate that the formation of the undersurface layer with the negative strain is not directly connected with the ballistic mechanisms related to ion mass. It can only be explained in terms of the influence of chemical properties of the ions on the formation of this layer. The model of the formation of such layer suggested for the case of implantation of $\mathrm{Si}$ with $\mathrm{Si}^{+}$ions ${ }^{2,5}$ cannot generally be attributed to all ions. As the magnitude of the negative strain is not connected with the mass of the ions ( $\mathrm{Mg}$ is heavier than $\mathrm{O}$ and $\mathrm{N}$ ), most probably formation of the damage is affected by the implanted species.

Since the deformation of the surface layer was shown to have a tetragonal character, the magnitude of the in-plane strain $\epsilon$ of the implanted layer caused by the thick undistorted substrate can be calculated from the measured $(\Delta d / d)_{\perp}$ as $^{11}$

$$
\epsilon=-\frac{(\Delta d / d)_{\perp}(1-\nu)}{(1+\nu)+(\Delta d / d)_{\perp}(1-\nu)},
$$

where $\nu$ is the Poisson ratio. The energy $E$ stored in the stressed crystal layer with the thickness $t$ due to the radiation damage is ${ }^{12}$ 


$$
E=\frac{Y}{1-\nu} \epsilon^{2} t
$$

where $Y$ is the Young's modulus. The highest estimated elastic energy was found to be stored in the layer implanted with $\mathrm{N}^{+}$ions at a dose of $2 \times 10^{17} \mathrm{~cm}^{-2}$. For this case the measured $(\Delta d / d)_{\perp}=1.4 \times 10^{-3}$. For $t=100 \mathrm{~nm}$ the value of $E$ is $1.1 \times 10^{-6} \mathrm{~J} / \mathrm{cm}^{2}$. This energy was transferred to the crystal lattice of the surface layer from the ions during implantation. The energy stored by the stressed crystal lattice is essentially different for the different ions implanted, while the energy deposited in nuclear collisions during implantation in such cases was approximately the same. This fact also confirms different mechanisms of defect-impurity interaction for different ion species. The difference in the stored energy may be connected to different binding energies of different $\mathrm{Si}$ impurity complexes. The strength of the chemical bonds (the bond dissociation energy) is $8.0 \mathrm{eV}$ for $\mathrm{O}-\mathrm{Si}$ and $4.6 \mathrm{eV}$ for $\mathrm{N}-\mathrm{Si}^{13} \mathrm{Ne}$ and $\mathrm{Mg}$ seem not to form stable bonds with $\mathrm{Si}$. However we assume that generation of the negative strain is not a direct result of the formation of Si-impurity complexes because the location of the negative strain does not follow the impurity profiles. We should note that the stored energy of the stressed crystal lattice may be transferred to impurities and defects and affect their behavior (diffusion, defect annealing, impurity electrical activation) during further technological processes. During annealing, with recovery of the crystal lattice in the surface layer, this energy is reduced.

The results obtained indicate that the implanted ions (oxygen and nitrogen) participate in the formation of the vacancy clusters in the undersurface region responsible for the negative strain observed. Most probably the efficiency of the formation of the vacancy clusters during high temperature implantation depends on the ability of the impurity atoms to form complexes with $\mathrm{Si}$ atoms. In the case of $\mathrm{Ne}^{+}$ implantation, the creation of precipitates (bubbles) is expected in the implanted layer which very likely prevents the formation of the layer with negative strain. The mechanism of the interaction between the $\mathrm{Mg}^{+}$ions and the damage is not clear yet, but the results obtained show that the formation of the damage depends essentially on the chemical properties of the implanted species.

\section{CONCLUSION}

The results of the investigation have shown that the general model of spatial separation of interstitials and vacancies based solely on the kinematics and not the chemistry of the ion-atom interaction suggested for the description of the damage formation at elevated temperatures for the case of $\mathrm{Si}^{+}$ions implanted into Si (Refs. 2 and 6) is not the only mechanism responsible for the formation of the two layers of distinct strain characteristics in the damage distribution. This statement is based on the fact that the irradiation with $\mathrm{Ne}^{+}$or $\mathrm{Mg}^{+}$does not produce such a damage distribution. Consequently, the implanted species very likely participate in the formation of the damage during implantation at elevated temperatures. Besides or instead of the spatial separation of the Frenkel pairs, the formation of precipitates should play an important role in damage development. Apart from this, the ion mass is of significant importance in the formation of the damage, but neither the negative strain at the surface nor the magnitude of the damage around the projected range is proportional to the ion mass. We conclude that a significant factor in the formation of extended defects and vacancy clusters is the chemical properties of the implanted species, their ability to precipitate and to form complexes with $\mathrm{Si}$ atoms.

\section{ACKNOWLEDGMENTS}

This research was partly supported by Fundação de Amparo à Pesquisa do Estado do Rio Grande do Sul (FAPERGS), Conselho Nacional de Desenvolvimento Científico e Tecnológico $(\mathrm{CNPq})$ and Financiadora de Estudos e Projetos (Finep).

${ }^{1}$ J. Linnros, R. G. Elliman, and W. L. Brown, J. Mater. Res. 3, 1208 (1988).

${ }^{2}$ O. W. Holland, J. D. Budai, and B. Nielsen, Mater. Sci. Eng., A 253, 240 (1998).

${ }^{3}$ A. E. White, K. T. Short, R. C. Dymes, J. M. Gibson, and R. Hull, Mater. Res. Soc. Symp. Proc. 107, 3 (1988).

${ }^{4}$ O. W. Holland, D. S. Zhou, and D. K. Thomas, Appl. Phys. Lett. 63, 896 (1993).

${ }^{5}$ O. W. Holland and C. W. White, Nucl. Instrum. Methods Phys. Res. B 59/60, 353 (1991)

${ }^{6}$ D. Venables and K. S. Jones, Nucl. Instrum. Methods Phys. Res. B 74, 65 (1993).

${ }^{7}$ D. Venables, K. S. Jones, F. Namavar, and J. M. Manke, Mater. Res. Soc. Symp. Proc. 235, 103 (1992).

${ }^{8}$ F. Eisen, in Channelling, edited by D. V. Morgan (Wiley, New York, 1973), p. 417.

${ }^{9}$ J. P. Ziegler, J. P. Biersack, and U. Littmark, The Stopping and Range of Ions in Solids (Pergamon, New York, 1985).

${ }^{10} \mathrm{O}$. W. Holland (private communication).

${ }^{11}$ Yu. Suprun-Belevich, F. Cristiano, A. Nejim, P. L. F. Hemment, and B. J. Sealy, Nucl. Instrum. Methods Phys. Res. B 140, 91 (1998).

${ }^{12}$ E. Kasper and F. Schäffler, Semicond. Semimet. 33, 223 (1991).

${ }^{13}$ Handbook of Material Science, edited by C. T. Lynch (Chemical Rubber, Cleveland, 1974), Vol. 1. 